\title{
From Machine Reading Comprehension to Dialogue State Tracking: Bridging the Gap
}

\author{
Shuyang Gao ${ }^{*, 1}$ Sanchit Agarwal $^{*, 1}$ Tagyoung Chung $^{1}$ Di Jin ${ }^{2}$ Dilek Hakkani-Tur ${ }^{1}$ \\ ${ }^{1}$ Amazon Alexa AI, Sunnyvale, CA, USA \\ ${ }^{2}$ Computer Science \& Artificial Intelligence Laboratory, MIT, MA, USA \\ \{shuyag, agsanchi, tagyoung, hakkanit\}@amazon.com,jindi15@mit.edu
}

\begin{abstract}
Dialogue state tracking (DST) is at the heart of task-oriented dialogue systems. However, the scarcity of labeled data is an obstacle to building accurate and robust state tracking systems that work across a variety of domains. Existing approaches generally require some dialogue data with state information and their ability to generalize to unknown domains is limited. In this paper, we propose using machine reading comprehension ( $\mathrm{RC}$ ) in state tracking from two perspectives: model architectures and datasets. We divide the slot types in dialogue state into categorical or extractive to borrow the advantages from both multiplechoice and span-based reading comprehension models. Our method achieves near the current state-of-the-art in joint goal accuracy on MultiWOZ 2.1 given full training data. More importantly, by leveraging machine reading comprehension datasets, our method outperforms the existing approaches by many a large margin in few-shot scenarios when the availability of in-domain data is limited. Lastly, even without any state tracking data, i.e., zero-shot scenario, our proposed approach achieves greater than $90 \%$ average slot accuracy in 12 out of 30 slots in MultiWOZ 2.1.
\end{abstract}

\section{Introduction}

Building a task-oriented dialogue system that can comprehend users' requests and complete tasks on their behalf is a challenging but fascinating problem. Dialogue state tracking (DST) is at the heart of task-oriented dialogue systems. It tracks the state of a dialogue during the conversation between a user and a system. The state is typically defined as the (slot_name, slot_value) pair that represents, given a slot, the value that the user provides or system-provided value that the user accepts.

\footnotetext{
*Authors contributed equally.
}

Despite the importance of DST in task-oriented dialogues systems, few large datasets are available. To address this issue, several methods have been proposed for data collection and bootstrapping the DST system. These approaches either utilize Wizard-of-Oz setup via crowd sourcing (Wen et al., 2017; Budzianowski et al., 2018) or Machines Talking To Machines (M2M) framework (Shah et al., 2018). Currently the most comprehensive dataset with state annotation is MultiWOZ (Budzianowski et al., 2018), which contains seven domains with around 10,000 dialogues. However, compared to other NLP datasets, MultiWOZ is still relatively small, especially for training data-intensive neural models. In addition, it is also a non-trivial to get a large amount of clean labeled data given the nature of task-oriented dialogues (Eric et al., 2019).

Another thread of approaches have tried to utilize data in a more efficient manner. These approaches (Wu et al., 2019; Zhou and Small, 2019) usually train the models on several domains and perform zero-shot or few-shot learning on unseen domains. However, these methods require slot definitions to be similar between the training data and the unseen test data. If such systems are given a completely new slot type, the performance would degrade significantly. Therefore, these approaches still rely on considerable amount of DST data to cover a broad range of slot categories.

We find machine reading comprehension task (RC) (Rajpurkar et al., 2016; Chen, 2018) as a source of inspiration to tackle these challenges. The RC task aims to evaluate how well machine models can understand human language, whose goals are actually similar to DST. Ultimately, DST focuses on the contextual understanding of users' request and inferring the state from the conversation, whereas RC focuses on the general understanding of the text regardless of its format, which can be either passages or conversations. In addition, 
recent advances have shown tremendous success in RC tasks. Thus, if we could formulate the DST task as a RC task, it could benefit DST in two aspects: first, we could take advantage of the fastgrowing $\mathrm{RC}$ research advances; second, we could make use of the abundant RC data to overcome the data scarcity issue in DST task.

Building upon this motivation, we formulate the DST task into an RC task by specially designing a question for each slot in the dialogue state, similar to Gao et al. (2019). Then, we divide the slots into two types: categorical and extractive, based on the number of slot values in the ontology. For instance, in MultiWOZ, slots such as parking take values of $\{$ Yes, No, Don't Care $\}$ and can thus be treated as categorical. In contrast, slots such as hotel-name may accept an unlimited number of possible values and these are treated as extractive. Accordingly, we propose two machine reading comprehension models for dialogue state tracking. For categorical slots, we use multiple-choice reading comprehension models where an answer has to be chosen from a limited number of options. And for the extractive dialogue state tracking, span-based reading comprehension are applied where the answer can be found in the form of a span in the conversation.

To summarize our approach and contributions:

- We divide the dialogue state slots into categorical and extractive types and use RC techniques for state tracking. Our approach can leverage the recent advances in the field of machine reading comprehension, including both multiple-choice and span-based reading comprehension models.

- We propose a two-stage training strategy. We first coarse-train the state tracking models on reading comprehension datasets, then finetune them on the target state tracking dataset.

- We show the effectiveness of our method under three scenarios: First, in full data setting, we show our method achieves close to the current state-of-the-art on MultiWoz 2.1 in terms of joint goal accuracy. Second, in fewshot setting, when only $1-10 \%$ of the training data is available, we show our methods significantly outperform the previous methods for 5 test domains in MultiWoz 2.0. In particular, we achieve $45.91 \%$ joint goal accuracy with just $1 \%$ (around 20-30 dialogues) of hotel domain data as compared to previous best result of $19.73 \%$ (Wu et al., 2019).
Thirdly, in zero-shot setting where no state tracking data is used for training, our models still achieve considerable average slot accuracy. More concretely, we show that 13 out of 30 slots in MultiWOZ 2.1 can achieve an average slot accuracy of greater than $90 \%$ without any training.

- We demonstrate the impact of canonicalization on extractive dialogue state tracking. We also categorize errors based on None and Not None slot values. We found the majority errors for our DST model come from distinguishing None or Not None for slots.

\section{Related Works}

Traditionally, dialogue state tracking methods (Liu and Lane, 2017; Mrkšić et al., 2016; Zhong et al., 2018; Nouri and Hosseini-Asl, 2018; Lee et al., 2019) assume a fully-known fixed ontology for all slots where the output space of a slot is constrained by the values in the ontology. However, such approaches cannot handle previously unseen values and do not scale well for slots such as restaurantname that can take potentially unbounded set of values. To alleviate these issues, Rastogi et al. (2017); Goel et al. (2018) generate and score slot-value candidates from the ontology, dialogue context $n$ grams, slot tagger outputs, or a combination of them. However, these approaches suffer if a reliable slot tagger is not available or if the slot value is longer than the candidate $n$-grams. $\mathrm{Xu}$ and $\mathrm{Hu}$ (2018) proposed attention-based pointing mechanism to find the start and end of the slot value to better tackle the issue of unseen slot values. Gao et al. (2019) proposed using a RC framework for state tracking. They track slot values by answering the question "what is the value of the slot?" through attention-based pointing to the dialogue context. Chao and Lane (2019); Rastogi et al. (2019) utilize BERT to encode the dialogue context and then point to slot-value span in the encoded context. Although these approaches are more practical and scalable, they suffer when the exact slot value does not appear in the context as expected by the backend database or if the value is not pointable. More recently, hybrid approaches have attempted to combine the benefits of both using predefined ontology (closed vocabulary) and dynamically generating candidate set or pointing (open vocabulary) approaches. Goel et al. (2019) select between the two approaches per slot based on dev set. Wu et al. 
(2019) utilize pointer generator network to either copy from the context or generate from vocabulary.

Perhaps, the most similar to our work is by Zhang et al. (2019) and Zhou and Small (2019) where they divide slot types into span-based (extractive) slots and pick-list (categorical) slots and use QA framework to point or pick values for these slots. A major limitation of these works is that they utilize heuristics to determine which slots should be categorical and which non-categorical. Moreover, in these settings most of the slots are treated as categorical (21/30 and 25/30), even though some of them have very large number of possible values, e.g., restaurant-name. This is not scalable especially when the ontology is large, not comprehensive, or when new domains/slots can occur at test time as in DSTC8 dataset (Rastogi et al., 2019).

There are recent efforts into building or adapting dialog state tracking systems in low source data scenarios Wu et al. (2019); Zhou and Small (2019). The general idea in these approaches is to treat all but one domain as in-domain data and test on the remaining unseen domain either directly (zero shot) or after fine-tuning on small percentage (1\%$10 \%$ ) of the unseen domain data (few shot). A major drawback of these approaches is that they require several labeled in-domain examples in order perform well on the unseen domain. This limits these approaches to in-domain slots and slot definitions and they do not generalize very well to new slots or completely unseen target domain. This also requires large amount of labeled data in the source domain, which may not be available in realworld scenario. Our proposed approach, on the other hand, utilizes domain-agnostic QA datasets with zero or a small percentage of DST data and significantly outperforms these approaches in lowresource settings.

\section{Methods}

\subsection{Dialogue State Tracking as Reading Comprehension}

Dialogue as Paragraph For a given dialogue at turn $t$, let us denote the user utterance tokens and the agent utterance tokens as $\mathbf{u}_{t}$ and $\mathbf{a}_{t}$ respectively. We concatenate the user utterance tokens and the agent utterance tokens at each turn to construct a sequence of tokens as $\mathbf{D}_{t}=\left\{\mathbf{u}_{1}, \mathbf{a}_{1}, \ldots, \mathbf{u}_{t}\right\}$. $\mathbf{D}_{t}$ can be viewed as the paragraph that we are going to ask questions on at turn $t$.
Slot as Question We can formulate a natural language question $\mathbf{q}_{i}$, for each slot $s_{i}$ in the dialogue state. Such a question describes the meaning of that slot in the dialogue state. Examples of (slot, question) pairs can be seen in Table 2 and 3. We formulate questions by considering characteristics of domain and slot. In this way, DST becomes finding answers $\mathbf{a}_{i}$ to the question $\mathbf{q}_{i}$ given the paragraph $\mathbf{D}_{t}$. Note that Gao et al. (2019) formulate dialogue state tracking problem in a similar way but their question formulation "what is the value of a slot?" is more abstract, whereas our questions are more concrete and meaningful to the dialogue.

\subsection{Span-based RC To Extractive DST}

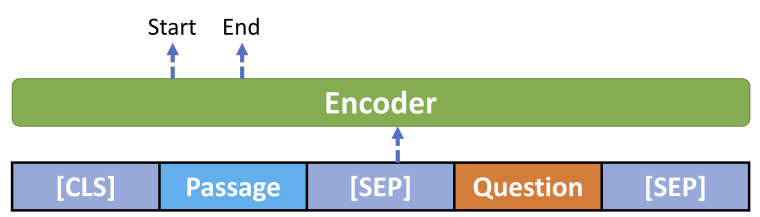

Figure 1: Model architecture for extractive state tracking. "Encoder"is a pre-trained sentence encoder such as BERT.

For many slots in the dialogue state such as names of attractions, restaurants, and departure times, one can often find their values in the dialogue context with exact matches. Slots with a wide range of values fits this description. Table 1 shows the exact match rate for each slot in MultiWOZ 2.1 dataset (Budzianowski et al., 2018; Eric et al., 2019) where slots with large number of possible values tend to have higher exact match rate ( $\geq 80 \%$ ). We call tracking such slots as extractive dialogue stack tracking (EDST).

This problem is similar to span-based RC where the goal is to find a span in the passage that best answers the question. Therefore, for EDST, we adopt the simple BERT-based question answering model used by Devlin et al. (2019), which has shown strong performance on multiple datasets ( $\mathrm{Ra}-$ jpurkar et al., 2016, 2018; Reddy et al., 2019). In this model as shown in Figure 1, the slot question and the dialogue are represented as a single sequence. The probability of a dialogue token $t_{i}$ being the start of the slot value span is computed as $p_{i}=\frac{e^{\mathbf{s} \cdot \mathbf{T}_{i}}}{\sum_{j} e^{\mathbf{s} \cdot \mathbf{T}_{j}}}$, where $\mathbf{T}_{j}$ is the embedding of each token $t_{j}$ and $\mathbf{s}$ is a learnable vector. A similar formula is applied for finding the end of the span.

Handling None Values At any given turn in the conversation, there are typically, many slots that 


\begin{tabular}{|c|c|c|c|c|}
\hline Slot Name & \# Possible Values & Exact Match Rate & Extractive & Categorical \\
\hline hotel.semi.type & 3 & $61.1 \%$ & $\times$ & $\checkmark$ \\
\hline hotel.semi.internet & 3 & $62.1 \%$ & $x$ & $\checkmark$ \\
\hline hotel.semi.parking & 4 & $63.1 \%$ & $x$ & $\checkmark$ \\
\hline restaurant.semi.pricerange & 4 & $97.8 \%$ & $\checkmark$ & $\checkmark$ \\
\hline hotel.semi.pricerange & 6 & $97.7 \%$ & $\checkmark$ & $\checkmark$ \\
\hline hotel.semi.area & 6 & $98.8 \%$ & $\checkmark$ & $\checkmark$ \\
\hline attraction.semi.area & 6 & $99.0 \%$ & $\checkmark$ & $\checkmark$ \\
\hline restaurant.semi.area & 6 & $99.2 \%$ & $\checkmark$ & $\checkmark$ \\
\hline hotel.semi.stars & 7 & $99.2 \%$ & $\checkmark$ & $\checkmark$ \\
\hline hotel.book.people & 8 & $98.2 \%$ & $\checkmark$ & $\checkmark$ \\
\hline hotel.book.stay & 8 & $98.9 \%$ & $\checkmark$ & $\checkmark$ \\
\hline train.semi.day & 8 & $99.3 \%$ & $\checkmark$ & $\checkmark$ \\
\hline restaurant.book.day & 8 & $98.7 \%$ & $\checkmark$ & $\checkmark$ \\
\hline restaurant.book.people & 8 & $99.1 \%$ & $\checkmark$ & $\checkmark$ \\
\hline hotel.book.day & 11 & $98.1 \%$ & $\checkmark$ & $\checkmark$ \\
\hline train.book.people & 12 & $94.7 \%$ & $\checkmark$ & $x$ \\
\hline train.semi.destination & 27 & $98.2 \%$ & $\checkmark$ & $\times$ \\
\hline attraction.semi.type & 27 & $86.6 \%$ & $\checkmark$ & $x$ \\
\hline train.semi.departure & 31 & $97.6 \%$ & $\checkmark$ & $x$ \\
\hline restaurant.book.time & 67 & $97.2 \%$ & $\checkmark$ & $x$ \\
\hline hotel.semi.name & 78 & $88.7 \%$ & $\checkmark$ & $x$ \\
\hline taxi.semi.arriveby & 97 & $91.9 \%$ & $\checkmark$ & $x$ \\
\hline restaurant.semi.food & 103 & $96.4 \%$ & $\checkmark$ & $x$ \\
\hline taxi.semi.leaveat & 108 & $81.1 \%$ & $\checkmark$ & $x$ \\
\hline train.semi.arriveby & 156 & $91.5 \%$ & $\checkmark$ & $x$ \\
\hline attraction.semi.name & 158 & $84.3 \%$ & $\checkmark$ & $x$ \\
\hline restaurant.semi.name & 182 & $93.9 \%$ & $\checkmark$ & $x$ \\
\hline train.semi.leaveat & 201 & $87.4 \%$ & $\checkmark$ & $x$ \\
\hline taxi.semi.destination & 251 & $87.9 \%$ & $\checkmark$ & $x$ \\
\hline taxi.semi.departure & 253 & $84.6 \%$ & $\checkmark$ & $x$ \\
\hline
\end{tabular}

Table 1: Slot statistics for MultiWOZ 2.1. We classify the slots into extractive or categorical based on their exact match rate in conversation as well as number of possible values. 3 slots are categorical only, 12 slots are both extractive and categorical, the remaining 15 slots are extractive only.

\begin{tabular}{l} 
Dialogue \\
\hline U: I'm so hungry. Can you find me a place to eat in the \\
city centre? \\
A: I'm happy to help! There are a great deal of restaurants \\
there. What type of food did you have in mind? \\
U: I do not care, it just needs to be expensive. \\
A: Fitzbillies restaurant serves British food would that be \\
okay? \\
U: Yes, may I have the address?
\end{tabular}

restaurant.semi.food: What type of food does the user want to eat?

Answer: [52-53] (I do not care, it just needs to be expensive)

restaurant.semi.name: What is the name of the restaurant where the user wants to eat?

Answer: [53-55] (Fitzbillies restaurant)

Table 2: Sample dialogue from MultiWOZ dataset showing framing of extractive DST to span-based RC. The span text (or don't care user utterance) is also shown in italics.

have not been mentioned or accepted yet by the user. All these slots must be assigned a None value in the dialogue state. We can view such cases as no answer exists in reading comprehension formulation. Similar to Devlin et al. (2019) for SQuAD 2.0 task, we assign the answer span with start and end at the beginning token [CLS] for these slots.

Handling Don't Care Values To handle don't care value in EDST, a span is also assigned to don't care in the dialogue. We find the dialogue turn when the slot value first becomes don't care and set the start and end of don't care span to be the start and end of the user utterance of this turn. See Table 2 for an example.

\subsection{Multiple-Choice Reading Comprehension to Categorical Dialogue State Tracking}

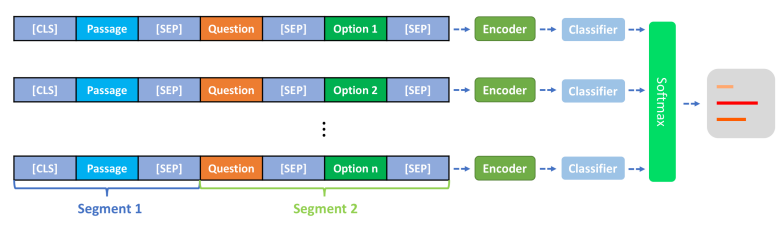

Figure 2: Model architecture for categorical dialog state tracking. "Encoder"is a pre-trained sentence encoder such as BERT. "Classifier" is a top-level fully connected layer.

The other type of slots in the dialogue state cannot be filled through exact match in the dialogue 


\begin{tabular}{l} 
Dialogue \\
\hline U: I am looking for a place to to stay that has cheap price \\
range it should be in a type of hotel \\
A: Okay, Do you have a specific area you want to stay in? \\
U: No, I just need to make sure it's cheap. Oh, and I need \\
parking. \\
hotel.semi.area: What is the area that the user wants to \\
book a hotel in? \\
A. East B. West C. North D. South E. Centre \\
F. Don't Care $\checkmark \quad$ G. Not Mentioned \\
hotel.semi.parking: Does the user want parking at the \\
hotel? \\
\begin{tabular}{l} 
A. Yes $\checkmark$ \\
\hline
\end{tabular}
\end{tabular}

Table 3: Sample dialogue from MultiWOZ dataset showing framing of categorical DST to multiple-choice RC.

context in a large number of cases. For example, a user might express intent for hotel parking as "oh! and make sure it has parking" but the slot hotelparking only accepts values from $\{Y e s, N o$, Don't Care $\}$. In this case, the state tracker needs to infer whether or not the user wants parking based on the user utterance and select the correct value from the list. These kind of slots may not have exact-match spans in the dialogue context but usually require a limited number of values to choose from.

Tracking these type of slots is surprisingly similar to multiple-choice reading comprehension (MCRC) tasks. In comparison to span-based RC tasks, the answers of MCRC datasets (Lai et al., 2017; Sun et al., 2019) are often in the form of open, natural language sentences and are not restricted to spans in text. Following the traditional models of MCRC (Devlin et al., 2019; Jin et al., 2019), we concatenate the slot question, the dialogue context and one of the answer choices into a long sequence. We then feed this sequence into a sentence encoder to obtain a logit vector. Given a question, we can get $m$ logit vectors assuming there are $m$ answer choices. We then transform these $m$ logit vectors into a probability vector through a fully connected layer and a softmax layer, see Figure 2 for details.

Handling None and Don't Care Values For each question, we simply add two additional choices "not mentioned" and "do not care" in the answer options, representing None and don't care, as shown in Table 3. It is worth noting that certain slots not only accept a limited number of values but also their values can be found as an exact-match span in the dialogue context. For these slots, both extractive and categorical DST models can be applied as shown in Table 1.

\section{Experiments}

\subsection{Datasets}

\begin{tabular}{lrr}
\hline & \# of passages & \# of examples \\
\hline MRQA (span-based) & 386,384 & 516,819 \\
DREAM (multi-choice) & 6,444 & 10,197 \\
RACE (multi-choice) & 27,933 & 97,687 \\
MultiWOZ & 8,420 & $298,978^{*}$ \\
\hline
\end{tabular}

Table 4: Statistics of datasets used. (*: we only report the number of positive examples (a non-empty value) in MultiWOZ for fair comparison.)

MultiWOZ We use the largest available multidomain dialogue dataset with state annotation: MultiWOZ 2.0 (Budzianowski et al., 2018) and MultiWOZ 2.1 (Eric et al., 2019), an enhanced, less noisier version of MultiWOZ 2.0 dataset, which contains 7 distinct domains across $10 \mathrm{~K}$ dialogues. We exclude hospital and police domain that have very few dialogues. This results in 5 remaining domains attraction, restaurant, taxi, train, hotel with a total of 30 (domain, slot) pairs in the dialog state following Wu et al. (2019); Zhang et al. (2019).

Reading Comprehension Datasets For spanbased RC dataset, we use the dataset from Machine Reading for Question Answering (MRQA) 2019 shared task (Fisch et al., 2019) that was focused on extractive question answering. MRQA contains six distinct datasets across different domains: SQuAD, NewsQA, TriviaQA, SearchQA, HotpotQA, and NaturalQuestions. In this dataset, any answer to a question is a segment of text or span in a given document. For multiple-choice RC dataset, we leverage the current largest multiplechoice QA dataset, RACE (Lai et al., 2017) as well as a dialogue-based multiple-choice QA dataset, DREAM (Sun et al., 2019). Both of these datasets are collected from English language exams that are carefully designed by educational experts to assess the comprehension level of English learners. Table 4 summarizes the statistics of datasets. It is worth noting that for MultiWOZ, although the number of examples are significantly more than multiple-choice QA datasets, the number of distinct questions are only 30 due to limited number of slot types.

\subsection{Canonicalization for Extractive Dialogue State Tracking}

For extractive dialogue state tracking, it is common that the model will choose a span that is either a 
super-set of the correct reference or has a similar meaning as the correct value but with a different wording. Following this observation, we adopt a simple canonicalization procedure after our spanbased model prediction. If the predicted value does not exist in the ontology of the slot, then we match the prediction with the value in the ontology that is closest to the predicted value in terms of edit distance $^{1}$. Note that this procedure is only applied at model inference time. At training time for extractive dialogue state tracking, the ontology is not required.

\subsection{Two-stage Training}

A two-stage training procedure is used to train the extractive and categorical dialogue state tracking models with both types of reading comprehension datasets (DREAM, RACE, and MRQA) and the dialogue state tracking dataset (MultiWOZ).

Reading Comprehension Training Stage For categorical dialogue state tracking model, we coarse-tune the model on DREAM and RACE. For extractive dialogue state tracking model, we coarsetune the model on MRQA dataset as a first step.

Dialog State Tracking Training Stage After being trained on the reading comprehension datasets, we expect our models to be capable of answering (passage, question) pairs. In this phase, we further fine-tune these models on the MultiWOZ dataset.

\section{Results and Analyses}

\subsection{DST with Full Training Data}

\begin{tabular}{l|c}
\hline \multicolumn{2}{c}{ Joint Goal Accuracy } \\
\hline SpanPtr (Xu and Hu, 2018) & $29.09 \%$ \\
\hline FJST (Eric et al., 2019) & $38.00 \%$ \\
\hline HyST (Goel et al., 2019) & $39.10 \%$ \\
\hline DSTreader (Gao et al., 2019) & $36.40 \%$ \\
\hline TRADE (Wu et al., 2019) & $45.96 \%$ \\
\hline DS-DST (Zhang et al., 2019) & $\mathbf{5 1 . 2 1} \%$ \\
\hline DSTQA w/span (Zhou and Small, 2019) & $49.67 \%$ \\
\hline DSTQA w/o span (Zhou and Small, 2019) & $51.17 \%$ \\
\hline STARC (this work) & $49.48 \%$ \\
\hline
\end{tabular}

Table 5: Joint Goal Accuracy on MultiWOZ 2.1 test set.

We use the full data in MultiWOZ 2.1 to test our models. For the first 15 slots with lowest number of possible values (from hotel.semi.type to ho-

\footnotetext{
${ }^{1}$ we use the function get_closest_matches of difflib in Python for this implementation.
}

tel.book.day in Table 1, we use our proposed categorical dialogue state tracking model whereas for the remaining 15 slots, we use the extractive dialogue state tracking model. We use the pre-trained word embedding RoBERTa-Large (Liu et al., 2019) in our experiment.

Table 5 summarizes the results. We can see that our model, STARC (State Tracking As Reading Comprehension), achieves close to the state-of-theart accuracy on MultiWOZ 2.1 in the full data setting. It is worth noting that the best performing approach DS-DST (Zhang et al., 2019), cherry-picks 9 slots as span-based slots whereas the remaining 21 slots are treated as categorical. Further, the second best result DSTQA w/o span (Zhou and Small, 2019) does not use span-based model for any slot. Unlike these state-of-the-art methods, our method simply categorizes the slots based on the number of values in the ontology. As a result, our approach uses less number of (15 as compared to 21 in DS-DST) and more reasonable (only those with few values in the ontology) categorical slots. Thus, our approach is more practical to be applied in a real-world scenario.

\begin{tabular}{l|c}
\hline Ablation & Dev Accuracy \\
\hline STARC (this work) & $\mathbf{5 3 . 9 5 \%}$ \\
- RC Coarse Tuning & $52.35 \%$ \\
- Canonicalization & $51.07 \%$ \\
- RC Coarse Tuning - Canonicalization & $50.84 \%$ \\
- Categorical Model & $47.86 \%$ \\
- Categorical Model - Canonicalization & $41.86 \%$ \\
\hline DS-DST Threshold-10 & $49.08 \%$ \\
DS-DST Span Only & $40.39 \%$ \\
\hline
\end{tabular}

Table 6: Ablation study with different aspects of our model and other comparable approaches. The numbers reported are joint goal accuracy on MultiWOZ 2.1 development set.

Ablation Study We also run ablation study to
understand which component of our model helps
with accuracy. Table 6 summarizes the results. For
fair comparison, we also report the numbers for DS-
DST Threshold-10 (Zhang et al., 2019) where they
also use the first 15 slots for categorical model and
the remaining for extractive model. We observe
that both two-stage training strategy using read-
ing comprehension data and canonicalization play
important role in higher accuracy. Without the cate-
gorical model (using extractive model for all slots),
STARC is still able to achieve joint goal accuracy
of $47.86 \%$. More interestingly, if we remove the
categorical model as well as the canonicalization,
the performance drops drastically, but is still slight 
better than purely extractive model of DS-DST.

\begin{tabular}{l|r|r}
\hline Error Type & Extractive & Categorical \\
\hline ref not none, predicted none & $43.7 \%$ & $31.4 \%$ \\
ref none, predicted not none & $25.6 \%$ & $58.4 \%$ \\
ref not none, predicted not none & $30.6 \%$ & $10.0 \%$ \\
\hline
\end{tabular}

Table 7: Type of errors made by each model.

Handling None Value Through error analysis of our models, we have learned that models' performance on None value has a significant impact on the overall accuracy. Table 7 summarizes our findings. We found that plurality errors for extractive model comes from cases where ground-truth is not None but model predicted None. For categorical model, the opposite was true. The majority errors were from model predicting not None value but the ground-truth is actually None. We leave further investigation on this issue as a future work.

\subsection{Few shot from RC to DST}

In few-shot setting, our model (both extractive and categorical) is pre-trained on reading comprehension datasets and we randomly select limited amount of target domain data for fine-tuning. We do not use out-of-domain MultiWOZ data for training for few-shot experiments unlike previous works. We evaluate our model with $1 \%, 5 \%$ and $10 \%$ of training data in the target domain. Table 8 shows the results of our model under this setting for five domains in MultiWOZ 2.0 $0^{2}$. We also report the few-shot results for other two models: TRADE (Wu et al., 2019) and DSTQA (Zhou and Small, 2019), where they perform the same few-shot experiments but pre-trained with a holdout strategy, i.e., training on the other four domains in MultiWOZ and fine-tune on the held-out domain. We can see that under all three different data settings, our model outperforms the TRADE and DSTQA models (expect the attraction domain for DSTQA) by a large margin. Especially in $1 \%$ data setting for hotel domain, which contains the most number of slots (10) among all the five domains, the joint goal accuracy dropped to $19.73 \%$ for TRADE while our model can still achieve relatively high joint goal accuracy of $45.91 \%$. This significant performance difference can be attributed to pre-training our models on reading comprehension datasets, which gives our model ability to comprehend passages or dialogues (which we have empiri-

\footnotetext{
${ }^{2}$ We are showing results on MultiWOZ 2.0 rather than 2.1 for the purpose of comparison to previous works.
}

cally verified in next section). The formulation of dialogue state tracking as a reading comprehension task helps the model to transfer comprehension capability. We also tried to repeat these experiments with vanilla pre-trained Roberta-Large model (without pretraining on RC dataset), but we could not even get these models to converge in such lowresource data settings. This further highlights the importance of RC pretraining for low resource dialogue state tracking.

\subsection{Zero shot from RC to DST}

In zero-shot experiments, we want to investigate how would the reading comprehension models behave on MultiWOZ dataset without any training on state tracking data. To do so, we train our models on reading comprehension datasets and test on MultiWOZ 2.1. Note that, in this setting, we only take labels in MultiWOZ 2.1 that are not missing, ignoring the data that is "None" in the dialogue state. For zero-shot experiments from multiple-choice $\mathrm{RC}$ to DST, we take the first fifteen slots in Table 1 that are classified as categorical. For zero shot from span-based RC to DST, we take twenty-seven slots which are extractive expect the first three slots in Table 1.

Figure 3 summarizes the results for hotel, restaurant, taxi and train domain in MultiWOZ 2.1. For attraction domain, please refer to the supplementary section A. We can see that most of the slots have an average accuracy of at least $50 \%$ or above in both multiple-choice RC and span-based RC approaches, indicating the effectiveness of RC data. For some slots such as hotel.stay, hotel.people, hotel.day, restaurant.people, restaurant.day, and train.day, we are able to achieve very high zeroshot accuracy (greater than 90\%). The zero-shot setting in TRADE (Wu et al., 2019), where the transfer is from the four source domains to the heldout target domain, fails completely on certain slot types like hotel.name. In contrast, our zero-shot experiments from RC to DST are able to transfer almost all the slots.

Table 9 illustrates the zero shot examples for span-based RC model. We can see that although the span-based RC model does not directly point to the state value itself, it usually points to a span that contains the ground truth state value and the canonicalization procedure then turns the span into the actual slot value. Such predicted spans can be viewed as evidence for getting the ground-truth 


\begin{tabular}{|c|c|c|c|c|c|c|c|c|c|c|c|c|c|c|c|}
\hline & \multicolumn{3}{|c|}{ Hotel } & \multicolumn{3}{|c|}{ Restaurant } & \multicolumn{3}{|c|}{ Attraction } & \multicolumn{3}{|c|}{ Train } & \multicolumn{3}{|c|}{ Taxi } \\
\hline & $1 \%$ & $5 \%$ & $10 \%$ & $1 \%$ & $5 \%$ & $10 \%$ & $1 \%$ & $5 \%$ & $10 \%$ & $1 \%$ & $5 \%$ & $10 \%$ & $1 \%$ & $5 \%$ & $10 \%$ \\
\hline TRADE & 19.73 & 37.45 & 41.42 & 42.42 & 55.70 & 60.94 & 35.88 & 57.55 & 63.12 & 59.83 & 69.27 & 71.11 & 63.81 & 66.58 & 70.19 \\
\hline DSTQA & N/A & 50.18 & 53.68 & N/A & 58.95 & 64.51 & N/A & 70.47 & 71.60 & N/A & 70.35 & 74.50 & N/A & 70.90 & 74.19 \\
\hline STARC & 45.91 & 52.59 & 57.37 & 51.65 & 60.49 & 64.66 & 40.39 & 65.34 & 66.27 & 65.67 & 74.11 & 75.08 & 72.58 & 75.35 & 79.61 \\
\hline
\end{tabular}

Table 8: Joint goal accuracy for few-shot experiments. Best numbers reported by TRADE and DSTQA are also shown.

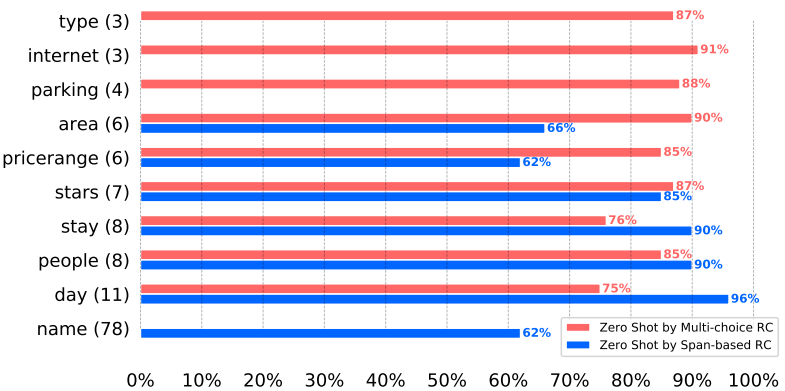

(a) Hotel

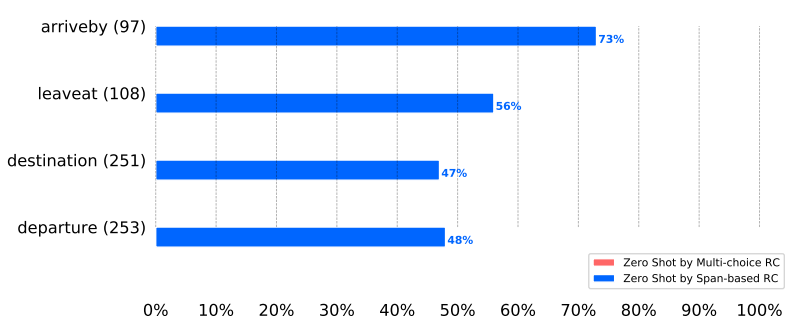

(c) Taxi

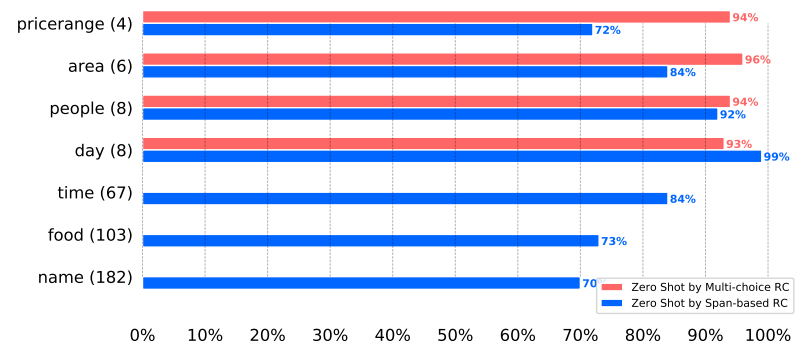

(b) Restaurant

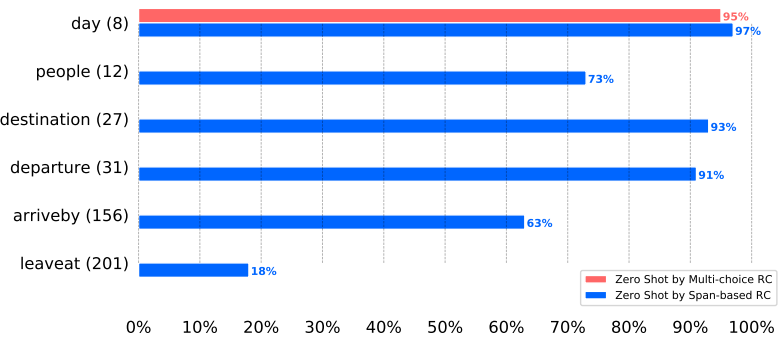

(d) Train

Figure 3: Zero-shot average slot accuracy using multi-choice and span-based RC to DST in hotel, restaurant, taxi, and train domain of MultiWOZ 2.1. The number in parentheses indicates the number of possible values that a slot can take.

\begin{tabular}{|c|c|}
\hline Example (Span-based RC model prediction is bolded) & Ground Truth State Value \\
\hline $\begin{array}{l}\text { Dialogue: “...A: sure, what area are you thinking of staying, } U: \boldsymbol{i} \text { do not have an area } \\
\text { preference but it needs to have free wifi and parking at a moderate price...." } \\
\text { Question: "which area is the hotel at?" (hotel.semi.area) }\end{array}$ & don't care \\
\hline $\begin{array}{l}\text { Dialogue: " } U: \text { i am looking for something fun to do on the east side of town . funky fun house } \\
\text { is my favorite place on the east side... } \\
\text { Question: "which area is the restaurant at?" (restaurant.semi.area) }\end{array}$ & east \\
\hline $\begin{array}{l}\text { Dialogue: "U: I need } 1 \text { that leaves after 13:30 for bishops stortford how about the tr8017? } \\
\text { A: it leaves at 15:29 and arrives at 16:07 in bishops stortford ...." } \\
\text { Question: "what time will the train leave from the departure location?" (train.semi.leaveat) }\end{array}$ & $15: 29$ \\
\hline $\begin{array}{l}\text { Dialogue: "U: hello } i \text { want to see some authentic architectures in cambridge!..." } \\
\text { Question: "what is the type of the attraction?" (attraction.semi.type) }\end{array}$ & architecture \\
\hline $\begin{array}{l}\text { Dialogue: "...A: can } i \text { help you with anything else? U: } i \text { would like to book a taxi from the } \\
\text { hong house to the hotel leaving by 10:15..." } \\
\text { Question: "where does the taxi leave from?" (taxi.semi.departure) }\end{array}$ & lan hong house \\
\hline
\end{tabular}

Table 9: Zero-shot examples to MultiWOZ 2.1 by span-based reading comprehension model trained on MRQA dataset. The predicted span by the span-based RC model are bolded.

dialogue state, which makes dialogue state tracking more explainable.

\section{Conclusion}

Task-oriented dialogue systems aim to help users to achieve a variety of tasks. It is not unusual to have hundreds of different domains in modern taskoriented virtual assistants. How can we ensure the 
dialogue system is robust enough to scale to different tasks given limited amount of data? Some approaches focus on domain expansion by training on several source domains and then adapting to the target domain. While such methods can be successful in certain cases, it is hard for them to generalize to other completely different out-of-domain tasks.

Machine reading comprehension provides us a clear and general basis for understanding the context given a wide variety of questions. By formulating the dialogue state tracking as reading comprehension, we can utilize the recent advances in reading comprehension models. More importantly, we can utilize reading comprehension datasets to mitigate some of the resource issues in task-oriented dialogue systems. As a result, we achieve much higher accuracy in dialogue state tracking across different domains given limited amount of data compared to the existing methods. As the variety of tasks and functionalities in a dialogue system continues to grow, general methods for tracking dialogue state across all tasks will become increasingly necessary. We hope that the developments suggested here will help to address this need.

\section{Acknowledgments}

We thank the anonymous reviewers for their insightful feedback that helped improve the paper.

\section{References}

Paweł Budzianowski, Tsung-Hsien Wen, Bo-Hsiang Tseng, Iñigo Casanueva, Ultes Stefan, Ramadan Osman, and Milica Gašić. 2018. Multiwoz - a largescale multi-domain wizard-of-oz dataset for taskoriented dialogue modelling. In Proceedings of the 2018 Conference on Empirical Methods in Natural Language Processing (EMNLP).

Guan-Lin Chao and Ian Lane. 2019. Bert-dst: Scalable end-to-end dialogue state tracking with bidirectional encoder representations from transformer. Proc. Interspeech 2019, pages 1468-1472.

Danqi Chen. 2018. Neural Reading Comprehension and Beyond. Ph.D. thesis, Stanford University.

Jacob Devlin, Ming-Wei Chang, Kenton Lee, and Kristina Toutanova. 2019. Bert: Pre-training of deep bidirectional transformers for language understanding. In Proceedings of the 2019 Conference of the North American Chapter of the Association for Computational Linguistics: Human Language Technologies, Volume 1 (Long and Short Papers), pages 4171-4186.
Mihail Eric, Rahul Goel, Shachi Paul, Abhishek Sethi, Sanchit Agarwal, Shuyang Gao, and Dilek HakkaniTur. 2019. Multiwoz 2.1: Multi-domain dialogue state corrections and state tracking baselines. arXiv preprint arXiv:1907.01669.

Adam Fisch, Alon Talmor, Robin Jia, Minjoon Seo, Eunsol Choi, and Danqi Chen. 2019. Mrqa 2019 shared task: Evaluating generalization in reading comprehension. arXiv preprint arXiv:1910.09753.

Shuyang Gao, Abhishek Sethi, Sanchit Agarwal, Tagyoung Chung, and Dilek Hakkani-Tur. 2019. Dialog state tracking: A neural reading comprehension approach. arXiv preprint arXiv:1908.01946.

Rahul Goel, Shachi Paul, Tagyoung Chung, Jeremie Lecomte, Arindam Mandal, and Dilek Hakkani-Tur. 2018. Flexible and scalable state tracking framework for goal-oriented dialogue systems. arXiv preprint arXiv:1811.12891.

Rahul Goel, Shachi Paul, and Dilek Hakkani-Tür. 2019. Hyst: A hybrid approach for flexible and accurate dialogue state tracking. arXiv preprint arXiv:1907.00883.

Di Jin, Shuyang Gao, Jiun-Yu Kao, Tagyoung Chung, and Dilek Hakkani-tur. 2019. Mmm: Multi-stage multi-task learning for multi-choice reading comprehension. arXiv preprint arXiv: 1910.00458.

Guokun Lai, Qizhe Xie, Hanxiao Liu, Yiming Yang, and Eduard Hovy. 2017. Race: Large-scale reading comprehension dataset from examinations. In Proceedings of the 2017 Conference on Empirical Methods in Natural Language Processing, pages 785794.

Hwaran Lee, Jinsik Lee, and Tae-Yoon Kim. 2019. Sumbt: Slot-utterance matching for universal and scalable belief tracking. arXiv preprint arXiv:1907.07421.

Bing Liu and Ian Lane. 2017. An end-to-end trainable neural network model with belief tracking for taskoriented dialog. arXiv preprint arXiv:1708.05956.

Yinhan Liu, Myle Ott, Naman Goyal, Jingfei Du, Mandar Joshi, Danqi Chen, Omer Levy, Mike Lewis, Luke Zettlemoyer, and Veselin Stoyanov. 2019. RoBERTa: A robustly optimized BERT pretraining approach. arXiv preprint arXiv:1907.11692.

Nikola Mrkšić, Diarmuid O Séaghdha, Tsung-Hsien Wen, Blaise Thomson, and Steve Young. 2016. Neural belief tracker: Data-driven dialogue state tracking. arXiv preprint arXiv:1606.03777.

Elnaz Nouri and Ehsan Hosseini-Asl. 2018. Toward scalable neural dialogue state tracking model. arXiv preprint arXiv:1812.00899.

Pranav Rajpurkar, Robin Jia, and Percy Liang. 2018. Know what you don't know: Unanswerable questions for squad. In Proceedings of the 56th Annual 
Meeting of the Association for Computational Linguistics (Volume 2: Short Papers), pages 784-789.

Pranav Rajpurkar, Jian Zhang, Konstantin Lopyrev, and Percy Liang. 2016. Squad: 100,000+ questions for machine comprehension of text. In Proceedings of the 2016 Conference on Empirical Methods in Natural Language Processing, pages 2383-2392.

Abhinav Rastogi, Dilek Hakkani-Tür, and Larry Heck. 2017. Scalable multi-domain dialogue state tracking. In 2017 IEEE Automatic Speech Recognition and Understanding Workshop (ASRU), pages 561568. IEEE.

Abhinav Rastogi, Xiaoxue Zang, Srinivas Sunkara, Raghav Gupta, and Pranav Khaitan. 2019. Towards scalable multi-domain conversational agents: The schema-guided dialogue dataset. arXiv preprint arXiv:1909.05855.

Siva Reddy, Danqi Chen, and Christopher D Manning. 2019. Coqa: A conversational question answering challenge. Transactions of the Association for Computational Linguistics, 7:249-266.

Pararth Shah, Dilek Hakkani-Tur, Bing Liu, and Gokhan Tur. 2018. Bootstrapping a neural conversational agent with dialogue self-play, crowdsourcing and on-line reinforcement learning. In Proceedings of the 2018 Conference of the North American Chapter of the Association for Computational Linguistics: Human Language Technologies, Volume 3 (Industry Papers), pages 41-51.

Kai Sun, Dian Yu, Jianshu Chen, Dong Yu, Yejin Choi, and Claire Cardie. 2019. Dream: A challenge data set and models for dialogue-based reading comprehension. Transactions of the Association for Computational Linguistics, 7:217-231.

TH Wen, D Vandyke, N Mrkšíc, M Gašíc, LM RojasBarahona, PH Su, S Ultes, and S Young. 2017. A network-based end-to-end trainable task-oriented dialogue system. In 15th Conference of the European Chapter of the Association for Computational Linguistics, EACL 2017-Proceedings of Conference, volume 1, pages 438-449.

Chien-Sheng Wu, Andrea Madotto, Ehsan HosseiniAsl, Caiming Xiong, Richard Socher, and Pascale Fung. 2019. Transferable multi-domain state generator for task-oriented dialogue systems. arXiv preprint arXiv:1905.08743.

Puyang Xu and Qi Hu. 2018. An end-to-end approach for handling unknown slot values in dialogue state tracking. arXiv preprint arXiv:1805.01555.

Jian-Guo Zhang, Kazuma Hashimoto, Chien-Sheng Wu, Yao Wan, Philip S Yu, Richard Socher, and Caiming Xiong. 2019. Find or classify? dual strategy for slot-value predictions on multi-domain dialog state tracking. arXiv preprint arXiv:1910.03544.
Victor Zhong, Caiming Xiong, and Richard Socher. 2018. Global-locally self-attentive dialogue state tracker. arXiv preprint arXiv:1805.09655.

Li Zhou and Kevin Small. 2019. Multi-domain dialogue state tracking as dynamic knowledge graph enhanced question answering. arXiv preprint arXiv:1911.06192. 


\section{A Zero shot experiments for Attraction domain in MultiWOZ 2.1}

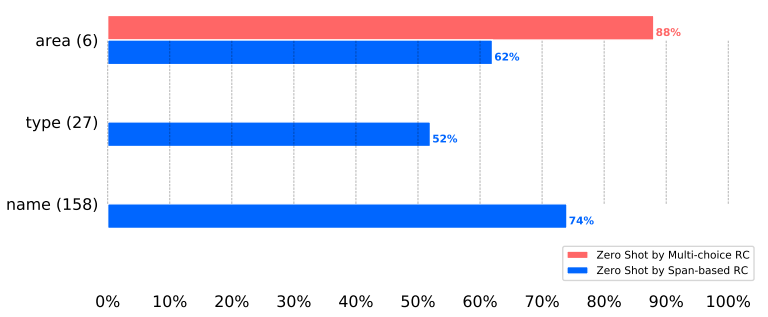

(a) Attraction

Figure 1: Zero shot average slot accuracy from RC to DST in attraction domain of MultiWOZ 2.1. The number within the brackets associated with each slot name in $y$-axis indicates the number of possible values that a slot can take.

\section{B Question Formation for Reading Comprehension}

The structural construct and the surface form of the question can have an impact on the performance of RC models. In this work, we handcrafted a question for each slot that needs to be tracked. Each question roughly asks What is the value of the slot that the user in interested in? The exact question was tailored to each specific slot also taking domains into account. We experimented with two sets of handcrafted questions. The first set was created in a procedural manner largely following a template. The other was created in a more freeform manner and was more natural. We did not notice any significant model performance difference between the two sets. However, we did not explore this dimension any further and leave it to future work. An interesting future direction could be to use a decoder to generate questions given slot description as the input. 